\title{
BECN1-knockout impairs tumor growth, migration and invasion by suppressing the cell cycle and partially suppressing the epithelial-mesenchymal transition of human triple-negative breast cancer cells
}

\author{
CHENG-LIN WU ${ }^{1 *}$, SHI-MENG ZHANG ${ }^{2 *}$, LI LIN $^{3}$, SHAN-SHAN GAO ${ }^{2}$, KAI-FEI FU ${ }^{1}$, \\ XIAO-DAN LIU ${ }^{2}$, YAN LIU ${ }^{1,4}$, LI-JUN ZHOU ${ }^{1}$ and PING-KUN ZHOU ${ }^{2}$ \\ ${ }^{1}$ Central Laboratory, Navy General Hospital, Beijing 100048; ${ }^{2}$ Department of Radiation Toxicology and Oncology, \\ Beijing Key Laboratory for Radiobiology, Beijing Institute of Radiation Medicine, Beijing 100850; \\ ${ }^{3}$ Department of Oncology, Peking University International Hospital, Beijing 102206; \\ ${ }^{4}$ The Third School of Clinical Medicine, Southern Medical University, Guangzhou, Guangdong 510630, P.R. China
}

Received January 15, 2018; Accepted June 5, 2018

DOI: $10.3892 /$ ijo.2018.4472

\begin{abstract}
Beclin1 (BECN1), which directly interacts with B-cell lymphoma 2, serves an important role in autophagy and is involved in the tumorigenesis of various types of cancer. However, the definite role of BECN1 in breast cancer remains controversial. Bi-allelic knockout of Becn1 in a mouse model leads to an embryonic lethal phenotype, which hampers further investigation. To generate cell lines with knockout of BECN1, the CRISPR/Cas9 technique was used to disrupt BECN1 in human triple-negative breast cancer (TNBC) MDA-MB-231 cells. To the best of our knowledge, the present study was the first to successfully disrupt BECN1 in MDA-MB-231 cells and to screen three stable monoclonal BECN1-knockout cell lines, suggesting that BECN1-knockout is not lethal in TNBC cells.
\end{abstract}

Correspondence to: Dr Li-Jun Zhou, Central Laboratory, Navy General Hospital, 6 Fucheng Road, Beijing 100048, P.R. China E-mail: hzzhoulj@126.com

Dr Ping-Kun Zhou, Department of Radiation Toxicology and Oncology, Beijing Key Laboratory for Radiobiology, Beijing Institute of Radiation Medicine, 27 Taiping Road, Beijing 100850, P.R. China

E-mail: zhoupk@bmi.ac.cn

"Contributed equally

Abbreviations: TNBC, triple-negative breast cancer; EMT, epithelialmesenchymal transition; iMMECs, immortalized mouse mammary epithelial cells; TSS, translation start site; T7EI, T7 Endonuclease I; CCK-8, Cell Counting Kit 8; WT, wild-type; KO, knockout

Key words: BECN1, CRISPR/Cas9, triple-negative breast cancer, growth, cell cycle, migration, invasion, epithelial-mesenchymal transition
Functional analysis revealed that complete loss of BECN1 suppressed MDA-MB-231 proliferation and colony formation via inducing $\mathrm{G}_{0} / \mathrm{G}_{1}$ cell cycle arrest, not apoptosis, in vitro. On the other hand, BECN1-knockout inhibited the migratory and invasive ability of MDA-MB-231 cells by partially reversing signals of epithelial-mesenchymal transition. Finally, analysis of publicly available gene expression datasets revealed increased expression of BECN1 in TNBC samples. Taken together, the results of the present study identified BECN1 as an oncogene, providing a novel potential target for the treatment of TNBC.

\section{Introduction}

Beclin1 (BECN1), a coiled-coil protein, directly interacts with B-cell lymphoma 2 (Bcl-2) (1). BECN1, also known as ATG6, belongs to the autophagy-related (Atg) family, key molecules in regulating autophagy $(2,3)$. BECN1 acts downstream of Ulk1 and protein kinase B (Akt) to initiate phagophore formation $(4,5)$.

BECN1 is monoallelically deleted in $40-75 \%$ of breast, ovarian and prostate cancer cases (6) and is most likely a haplo-insufficient tumor suppressor (7-9). BECN1 has the potential to predict a favorable prognosis in gastric cancer, breast cancer, and lymphoma (10). However, the exact role of $B E C N 1$ in breast cancer is not fully understood. Monoallelic loss of Becnl may promote breast cancer development in Wntl-driven tumorigenesis, suggesting a tumor suppressing role of BECN1 (11). By contrast, monoallelic deletion of Becnl promotes the formation of spontaneous lung cancer, liver cancer and lymphomas, but only led to mammary hyperplasia in C57BL/6J mice (8) and does not affect ERBB2- and PyMTdriven mammary tumorigenesis in immortalized mouse mammary epithelial cells (iMMECs) (12). Furthermore, monoallelic Becn1 loss inhibits Palb2-associated mammary tumorigenesis in a Trp53-wild-type background (13). Gong et al (14) also demonstrated that BECN1 promotes 
tumorigenicity in breast cancer stem-like cells. These seemingly paradoxical roles of BECN1 in breast cancer progression may be explained by various cellular contexts resulting in different consequences. In addition, bi-allelic knockout of Becnl leads to embryolethality in a mouse model, hindering further investigation (9). To further investigate the function of BECN1 in breast cancer, the present study used the CRISPR/Cas9 system to disrupt the BECN1 gene in the human triple-negative breast cancer (TNBC) MDA-MB-231 cell line. Since being improved to edit mammalian genes (15), CRISPR/Cas9 has been widely used to edit genes in numerous species (16-18).

In the present study, BECN1 was successfully disrupted in MDA-MB-231 cells and three stable monoclonal $B E C N 1$-knockout cell lines were screened for the first time. The MDA-MB-231 cells with BECN1-knockout exhibited markedly decreased proliferation, colony formation, migration and invasion accompanied by $\mathrm{G}_{0} / \mathrm{G}_{1}$ cell cycle arrest and a partial epithelial-mesenchymal transition (EMT) signal reversal in vitro. On the other hand, it was demonstrated that BECNI expression was increased in TNBC samples. Therefore, the results of the present study provide solid evidence for the oncogenic role of $B E C N 1$ in TNBC growth, migration and invasion.

\section{Materials and methods}

Cell culture. The human TNBC MDA-MB-231 cell line, human epithelial breast cancer BT-474 cell line and human embryonic kidney 293T and 293A cell lines were purchased from the American Type Culture Collection (Manassas, VA, USA), and were cultured in Dulbecco's modified Eagle's medium (DMEM), supplemented with $10 \%$ fetal bovine serum (FBS; both Thermo Fisher Scientific, Inc., Waltham, MA, USA) at $37^{\circ} \mathrm{C}$ in a humidified $5 \% \mathrm{CO}_{2}$ atmosphere.

Construction of plasmids. Three G(N19)NGG sequences downstream from the BECN1 TSS were selected as potential targeting sites. $\mathrm{N}$ indicates any nucleotide (A, T, G or $\mathrm{C}$ ). The three targeting sites were as follows: B432, GTCCAACAAC AGCACCATGCAGG; B497, GGACACGAGTTTCAAGAT CCTGG; and B525, GTCACCATCCAGGAACTCACAGG. To construct B432, B497 and B525 sgRNA lentiviral plasmids, respective primer pairs were used as a template for CRIF and CRIR PCR amplification in separate reactions (Table I). The PCR product was inserted into the pLKO.1 plasmid (Sigma-Aldrich; Merck KGaA, Darmstadt, Germany) using Gibson master mix (New England Biolabs, Inc., Ipswich, MA, USA). The flag-tagged Cas9 gene was cloned into the pShuttle-CMV plasmid (a gift from Dr Pu-Min Zhang) at the $B g l \mathrm{II}$ and $\mathrm{XhoI}$ restriction sites. Following linearizing with PmeI, pShuttle-CMV-Cas9 was co-transformed with the pAdEasy-1 plasmid (a gift from Dr Pu-Min Zhang) into BJ5183 cells (Stratagene; Agilent Technologies, Inc., Santa Clara, CA, USA), according to the manufacturer's protocol. Following incubation overnight at $37^{\circ} \mathrm{C}$, positive clones selected on LB plates containing $50 \mu \mathrm{g} / \mathrm{ml}$ kanamycin (Sigma-Aldrich; Merck KGaA) were identified by digesting with PacI.

CRISPR efficiency evaluation. The UCA ${ }^{\mathrm{TM}}$ CRISPR efficiency evaluation kit was purchased from Beijing Biocytogen Co.,
Ltd. (Beijing, China). Briefly, the targeting sequence of BECN1 amplified with the primer set B371_F and B793_R (Table I) was cloned into a precut pUCA plasmid (UCA-BECN1; Beijing Biocytogen Co., Ltd.). The UCA-BECN1, sgRNA and Flag-Cas9 plasmids were co-transfected into 293T cells using Lipofectamine ${ }^{\mathrm{TM}} 3000$ (Thermo Fisher Scientific, Inc.). Approximately $48 \mathrm{~h}$ after transfection, cells were lysed and luciferase activity was measured using the dual-luciferase reporter assay (Promega Corporation, Madison, WI, USA) with a Lumat LB 9501 Luminometer (Berthold Technologies GmbH \& Co., Bad Wildbad, Germany). CRISPR efficiency was calculated as the ratio of firefly luciferase to Renilla luciferase activity.

Lentivirus and adenovirus packaging and infection. To package the sgRNA lentivirus, the sgRNA plasmid and packaging plasmids, pMD and SPA were co-transfected into 293T cells as described earlier. The supernatant was harvested $48 \mathrm{~h}$ after transfection and used to infect MDA-MB-231 cells. The recombinant Ad plasmid was linearized with $P a c$ I and transfected into 293A cells using Lipofectamine ${ }^{\mathrm{TM}} 3000$ (Thermo Fisher Scientific, Inc.) to produce Cas9 adenovirus. Infected MDA-MB-231 cells were screened for sgRNA-positive cells by adding $2 \mu \mathrm{g} / \mathrm{ml}$ puromycin (Sigma-Aldrich; Merck $\mathrm{KGaA}$ ) for one week. Puromycin-resistant cells were further infected with Cas9 adenovirus for $12 \mathrm{~h}$. After 4 days of culture, the cells were well prepared for the subsequent experiments.

Cell genomic DNA extraction and target sequence amplification. The genomic DNA of MDA-MB-231 cells was extracted using a Qiagen Genomic kit (Qiagen GmbH, Hilden, Germany). Next, the genomic region surrounding the CRISPR/Cas9 target site was amplified by PCR using the primer set, B371_F and B793_R (Table I). PCR was performed using GoTaq ${ }^{\circledR}$ Green Master mix (Promega Corporation) in an ABI 2720 Thermal cycler (Applied Biosystems; Thermo Fisher Scientific, Inc.) as follows: $95^{\circ} \mathrm{C}$ for $2 \mathrm{~min} ; 33$ cycles at $95^{\circ} \mathrm{C}$ for $30 \mathrm{sec}, 60^{\circ} \mathrm{C}$ for $30 \mathrm{sec}$ and $72^{\circ} \mathrm{C}$ for $30 \mathrm{sec}$; and $72^{\circ} \mathrm{C}$ for $5 \mathrm{~min}$. The 423-bp PCR product was verified using $1.5 \%$ agarose gel electrophoresis and then purified using the QIAquick ${ }^{\circledR}$ gel extraction kit (Qiagen $\mathrm{GmbH})$.

T7 Endonuclease I (T7EI) digestion assay. T7EI was purchased from NEB. Prior to T7EI digestion, the PCR product amplified using the primer set, B371_F and B793_R (Table I), was denatured and annealed. In brief, the PCR product was incubated in $94^{\circ} \mathrm{C}$ water and cooled to room temperature naturally. The PCR product was then digested with T7EI at $37^{\circ} \mathrm{C}$ for $2 \mathrm{~h}$ and resolved with $1.5 \%$ agarose gel electrophoresis.

Sequencing analysis of a single cell colony. The genomic DNA of a single cell colony was extracted using a Qiagen Genomic kit (Qiagen $\mathrm{GmbH}$ ) and amplified using the primer set, B371_F and B793_R (Table I). Following purification, the PCR product was ligated into the pGEM-T Easy vector (Promega Corporation). The ligation product was used to transform competent $E$. coli DH5 $\alpha$ (Thermo Fisher Scientific, Inc.), and positive clones selected on LB plates containing $100 \mu \mathrm{g} / \mathrm{ml}$ ampicillin (Sigma-Aldrich; Merck KGaA) were sequenced using the universal $\mathrm{T} 7$ primer. 
Table I. Oligonucleotides used in the present study.

\begin{tabular}{|c|c|}
\hline Oligonucleotide & Sequence \\
\hline B432_F & 5'-GAAAGGACGAAACACCGTC CAACAACAGCACCATGCGTTTTAGAGCTAGAAAT-3' \\
\hline B432_R & 5'-ATTTCTAGCTCTAAAACGCA TGGTGCTGTTGTTGGACGGTGTTTCGTCCTTTC-3' \\
\hline B497_F & 5'-GAAAGGACGAAACACCGGAC ACGAGTTTCAAGATCCGTTTTAGAGCTAGAAAT-3' \\
\hline B497_R & 5'-ATTTCTAGCTCTAAAACGGAT CTTGAAACTCGTGTCCGGTGTTTCGTCCTTTC-3' \\
\hline B525_F & 5'-GAAAGGACGAAACACCGTC ACCATCCAGGAACTCACGTTTTAGAGCTAGAAAT-3' \\
\hline B525_R & 5'-ATTTCTAGCTCTAAAACGTGAG TTCCTGGATGGTGACGGTGTTTCGTCCTTTC-3' \\
\hline CRI_F & 5'-GTATTTCGATTTCTTGGCTTTATA TATCTTGTGGAAAGGACGAAACACCG-3' \\
\hline CRI_R & 5'-GTTGATAACGGACTAGCCTTATT TTAACTTGCTATTTCTAGCTCTAAAAC-3' \\
\hline B371_F & 5'-GGCGTCGCTTCTCCCTAATGTTG-3' \\
\hline B793_R & 5'-GCCCGCCTCGGTCTCCCAAAATGC-3' \\
\hline N-cadherin_F & 5'-AGCCAACCTTAACTGAGGAGT-3' \\
\hline N-cadherin_R & 5'-GGCAAGTTGATTGGAGGGATG-3' \\
\hline Vimentin_F & 5'-CGAAAACACCCTGCAATCTT-3' \\
\hline Vimentin_R & 5'-CTGGATTTCCTCTTCGTGGA-3' \\
\hline Claudin-1_F & 5'-GTCATTGGGGGTGCGATA-3' \\
\hline Claudin-1_R & 5'-GGTGTTGGGTAAGAGGTTGTTT-3' \\
\hline Snail_F & 5'-CCTGCGTCTGCGGAACCT-3' \\
\hline Snail_R & 5'-CCTGGCACTGGTACTTCTTGACA-3' \\
\hline GAPDH_F & 5'-ACGGATTTGGTCGTATTGGG-3' \\
\hline GAPDH_R & 5'-TGATTTTGGAGGGATCTCGC-3' \\
\hline E-cadherin_F & 5'-GCCTCCTGAAAAGAGAGTGGAAG-3' \\
\hline E-cadherin_R & 5'-TGGCAGTGTCTCTCCAAATCCG-3' \\
\hline
\end{tabular}

Western blotting. Cells were lysed using radioimmunoprecipitation assay buffer (Applygen Technologies Inc., Beijing, China) supplemented with protease and phosphatase inhibitors (Roche Diagnostics GmbH, Mannheim, Germany). The concentration of the protein was determined by Pierce ${ }^{\mathrm{TM}}$ bicinchoninic acid protein assay kit (Thermo Fisher Scientific, Inc.). Protein lysate (20 $\mu$ g per lane) was separated by $10 \%$ SDS-PAGE gel and transferred onto nitrocellulose membranes (EMD Millipore, Billerica, MA, USA). The membranes were blocked with 5\% skimmed milk (BD Biosciences, San Jose, CA, USA) in TBST for $2 \mathrm{~h}$ at room temperature and incubated overnight at $4^{\circ} \mathrm{C}$ with the following primary antibodies: BECN1 (dilution, 1:2,000; cat. no. sc-11427; Santa Cruz Biotechnology, Inc., Dallas, TX, USA), GAPDH (dilution, 1:2,000; cat. no. sc-365062; Santa Cruz Biotechnology, Inc.), N-cadherin (dilution, 1:1,000; cat. no. 13116; Cell Signaling Technology, Inc., Danvers, MA, USA), Snail (dilution, 1:1,000; cat. no. 3879; Cell Signaling Technology, Inc.), Claudin-1 (dilution, 1:1,000; cat. no. 13255; Cell Signaling Technology, Inc.), E-cadherin (dilution, 1:1,000; cat. no. 3195; Cell Signaling Technology, Inc.) and Vimentin (dilution, 1:8,000; cat. no. ab92547; Abcam, Cambridge, UK). Next, the membranes were incubated with horseradish peroxidase-conjugated antirabbit or anti-mouse secondary antibodies (dilution, 1:5,000; cat. no. 7074 and 7076; Cell Signaling Technology, Inc.) for $1 \mathrm{~h}$ at room temperature. Bands were visualized using an enhanced chemiluminescence reagent (EMD Millipore).

Reverse transcription-quantitative polymerase chain reaction ( $q P C R)$. Total RNA was isolated using TRIzol reagent
(Thermo Fisher Scientific, Inc.). Next, reverse transcription of $2 \mu \mathrm{g}$ total RNA was performed using Superscript ${ }^{\mathrm{TM}}$ III transcriptase (Thermo Fisher Scientific, Inc.). Regarding qPCR, cDNA was amplified using THUNDERBIRD SYBR qPCR mix (Toyobo Life Science, Osaka, Japan) in a Chromo4 ${ }^{\mathrm{TM}}$ system (Bio-Rad Laboratories, Inc., Hercules, CA, USA). PCR started with a 10 -min denaturation step at $95^{\circ} \mathrm{C}$, followed by 40 step cycles at $95^{\circ} \mathrm{C}$ for $15 \mathrm{sec}$ and $60^{\circ} \mathrm{C}$ for $60 \mathrm{sec}$. The expression of $\mathrm{N}$-cadherin, Vimentin, Claudin-1, Snail and E-cadherin was analyzed using the primers shown in Table I. GAPDH was used as an internal control for normalization. The $2^{-\Delta \Delta \mathrm{Cq}}$ method was used to calculate the relative expression levels (19).

Cell Counting kit 8 (CCK-8) and colony formation assays. For the CCK-8 assay, MDA-MB-231 cells (1,000 cells/ well) were seeded into 96 -well plates and incubated for 7 consecutive days. CCK-8 assays were performed in quintuplicate and proliferation of cells was measured by adding $10 \mu 1$ CCK-8 reagent (Dojindo Molecular Technologies, Inc., Kumamoto, Japan) to each well at different time-points. Plates were incubated at $37^{\circ} \mathrm{C}$ for $2.5 \mathrm{~h}$ and absorbance was evaluated using a microplate reader (Bio-Rad Laboratories, Inc.) at $450 \mathrm{~nm}$.

For the colony formation assay, MDA-MB-231 cells $(1,000$ cells/well $)$ were plated into 6 -well plates. Cells were cultured for up to 14 days and fresh DMEM supplemented with 10\% FBS (both Thermo Fisher Scientific, Inc.) was changed every 3 days. Cells were fixed in paraformaldehyde for $10 \mathrm{~min}$ 
at room temperature and stained with crystal violet for $15 \mathrm{~min}$ at room temperature. Colonies with $>50$ cells were counted.

Flow cytometric analysis. For the apoptosis assay, cells were stained using the PE Annexin V Apoptosis Detection kit I (BD Biosciences), according to the manufacturer's protocol. In brief, cells were digested with trypsin without EDTA, washed two times with PBS and resuspended in $100 \mu \mathrm{l}$ binding buffer, followed by simultaneous staining with $5 \mu \mathrm{l}$ PE Annexin V and $5 \mu \mathrm{l}$ 7-AAD for $15 \mathrm{~min}$ at room temperature in the dark, and then analyzed using a flow cytometer (BD Biosciences).

For cell cycle analysis, cells were harvested and fixed in cold $70 \%$ alcohol at $4^{\circ} \mathrm{C}$ overnight. Cells were collected by centrifugation ( $800 \mathrm{x} \mathrm{g}$ for $5 \mathrm{~min}$ at room temperature), washed twice in PBS and incubated with FxCycle ${ }^{\mathrm{TM}} \mathrm{PI} / \mathrm{RNase}$ Staining Solution (Thermo Fisher Scientific, Inc.) for $30 \mathrm{~min}$ at room temperature in the dark. Cell cycle distribution was then evaluated using a flow cytometer and CellQuest ${ }^{\mathrm{TM}}$ software (version 6.0; BD Biosciences).

Wound healing assay. For the wound healing assay, cells were seeded into 6-well plates and an artificial wound was made using a sterile $200 \mu \mathrm{l}$ pipette tip. Following rinsing with PBS, cells were incubated in serum-free DMEM (Thermo Fisher Scientific, Inc.). Images of the scratch gap were captured at 0 and $24 \mathrm{~h}$ under a light microscope (magnification, x100; Eclipse Ti-U; Nikon Corporation, Tokyo,Japan). The percentage of wound healing was evaluated as follows: [1- (width of the wound at $24 \mathrm{~h} /$ width of the wound at $0 \mathrm{~h}$ )] $\times 100$.

Transwell migration and invasion assays. Transwell chambers (8 $\mu \mathrm{m}$-pore size; Corning Incorporated, Corning, NY, USA) coated with or without diluted Matrigel (BD Biosciences) on the upper chamber were used for invasion or migration assays. Briefly, 5x10 ${ }^{4}$ MDA-MB-231 cells in serum-free DMEM (Thermo Fisher Scientific, Inc.) were seeded into the upper chamber and medium supplemented with $10 \%$ FBS was added to the lower chamber. Following culture for 8 or $24 \mathrm{~h}$, cells remaining on the upper side of the filter membrane were gently wiped off using a cotton swab, while cells that migrated or invaded the lower chamber were fixed with paraformaldehyde for $10 \mathrm{~min}$ at room temperature and stained with crystal violet for $15 \mathrm{~min}$ at room temperature. Cells were counted in five different randomly selected fields under a light microscope (magnification, x100; Nikon Corporation).

GEO database analysis. Gene expression data (accession nos., GSE81838 and GSE65194) were retrieved from the NCBI Gene Expression Omnibus (GEO) database. Details of patients and analysis of microarray data were as previously described $(20,21)$. GSE81838 consisted of 10 paired TNBC tissues and adjacent normal stromal tissues, and GSE65194 contained 41 TNBC samples and 11 normal breast tissue samples. Expression data from GSE81838 were normalized using the Robust MultiChip Averaging (RMA) algorithm implemented in the Bioconductor package Affy and $\log 2$-transformed. Data from GSE65194 were analyzed using standard AffyCDF or Brainarray HGU133Plus2_Hs_ ENTREZG version 13 custom chipset definition file and normalized using GeneChip RMA (GC-RMA).
Statistical analysis. SPSS version 19.0 software (IBM Corp., Armonk, NY, USA) was used for statistical analyses. Data are presented as the mean \pm standard deviation (SD) and error bars represent SD values from three independent experiments, unless otherwise indicated. Multiple-group comparisons were performed using one-way analysis of variance, followed by the Bonferroni's post hoc test. Comparisons between two groups were calculated using the two-sample t-test. $\mathrm{P}<0.05$ was considered to indicate a statistically significant difference.

\section{Results}

Establishing BECN1-knockout MDA-MB-231 cell lines. Becnl bi-allelic knockout mice die early in embryogenesis (9), which hampers further investigation of the functions of $B E C N 1$ in autophagy and tumorigenesis. In order to determine if cells survive following BECN1-knockout, the CRISPR/ Cas9 technique was used to establish a BECN1-knockout cell line. Using the free online bioinformatical sgRNA design tool (http://crispr.mit.edu/) developed by Dr Feng Zhang, the genomic and cDNA sequences downstream of the translation start site (TSS) were analyzed. As demonstrated in Fig. 1A, three sequences with the highest scores were selected to construct sgRNA lentivirus vectors. In order to assess vector efficiency, a luciferase reporter assay was performed (Fig. 1B).

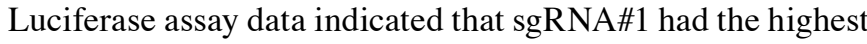
efficiency, which was also confirmed by western blot analysis (Fig. 1C).

Based on the aforementioned results, sgRNA\#1 was selected to package the lentivirus that was used to infect MDA-MB-231 cells. Puromycin-resistant cells were further infected with Cas9 adenovirus. Following serial dilution and culturing for up to two weeks, $>20$ single cell colonies were subjected to T7 Endonuclease I (T7EI) digestion. Five clones were subjected to a second round of T7EI digestion (Fig. 2A). Clones A, B and D (renamed KO\#1, KO\#2 and

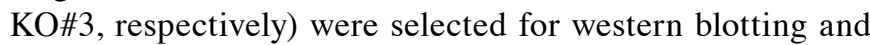
sequencing verification. Western blotting verified that BECN1 was not expressed in these three clones (Fig. 2B). Sequencing indicated that the loss of BECN1 expression was due to multiple InDels occurring at the BECN1 locus, all of which led to frame shifts and subsequent gene knockout (Fig. 2C). Taken together, the results indicated that stable cell lines that could survive following BECN1-knockout were successfully constructed.

BECN1-knockout inhibits proliferation and colony formation of MDA-MB-231 cells via inducing cell cycle arrest, not apoptosis. Since MDA-MB-231 cells with BECN1-knockout were able to survive, the effect of BECN1-knockout on cell growth was analyzed by comparing the viability of BECN1-knockout and wild-type (WT) MDA-MB-231 cells between 24 and $168 \mathrm{~h}$ after inoculation. As demonstrated in Fig. 3A, no significant difference in the viability of BECN1-knockout MDA-MB-231 cells was observed when compared with WT cells at $24 \mathrm{~h}$. However, when compared with WT MDA-MB-231 cells, $B E C N 1 \mathrm{KO \# 1}$ cells exhibited markedly decreased proliferation after $72 \mathrm{~h}$, and the proliferation of BECN1 KO\#2 and $\mathrm{KO \# 3}$ cells was significantly inhibited after $48 \mathrm{~h}$ in culture $(\mathrm{P}<0.001)$. The colony formation assay is an in vitro 
A
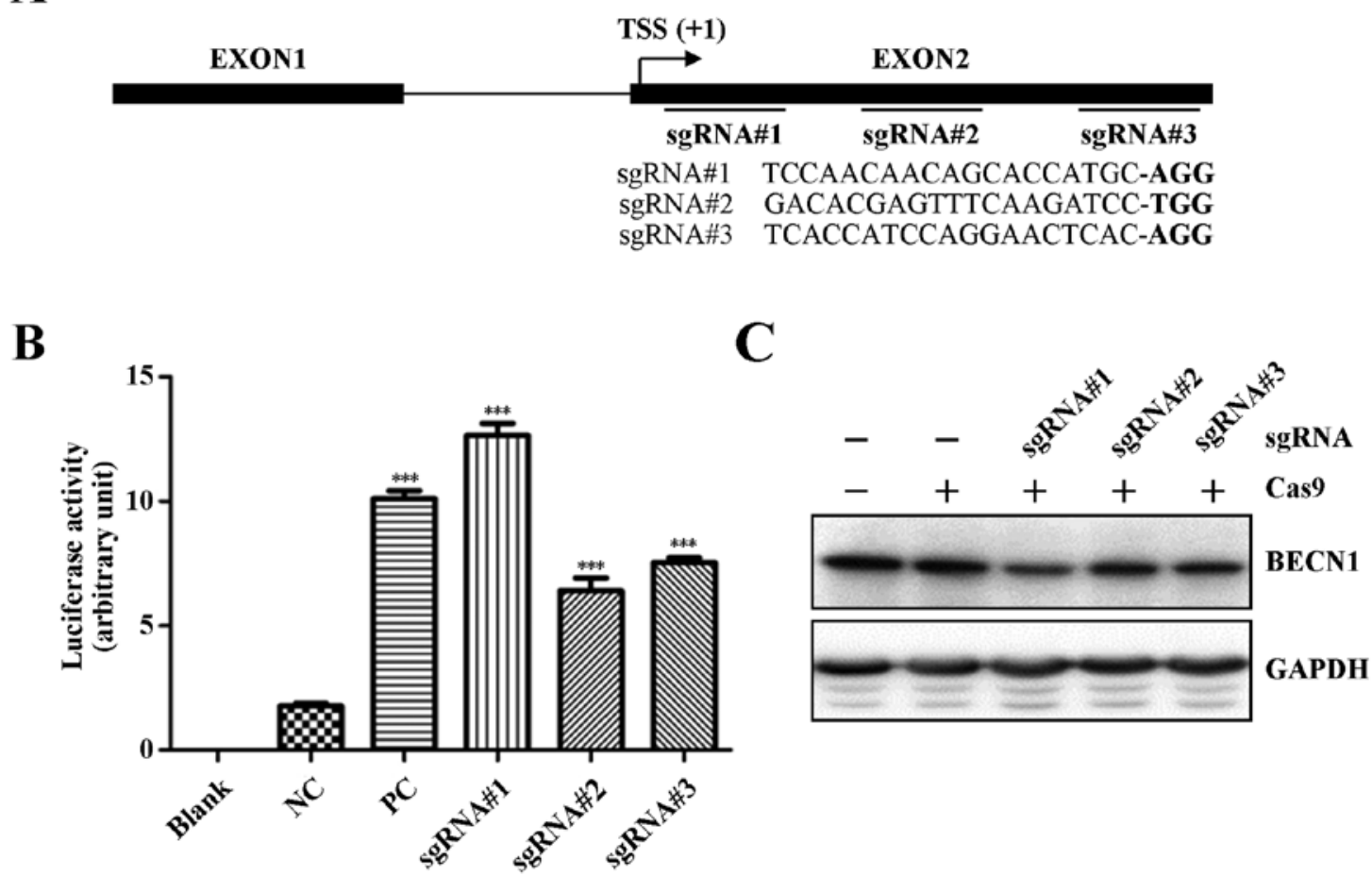

Figure 1. sgRNA-directed BECN1 gene editing efficiency assay. (A) A schematic representation of three different sgRNAs targeting EXON2 of BECN1. TSS , translation start site; black shading block, exon; black line, intron. (B) Luciferase reporter assay. The DNA fragment comprising the sgRNA targeting site with no mutation was cloned into the pUCA-LUC reporter plasmid (UCA-BECN1). The UCA-BECN1, sgRNA and Flag-Cas9 plasmids were co-transfected into $293 \mathrm{~T}$ cells, and luciferase activities were measured $48 \mathrm{~h}$ post-transfection. NC, negative control; PC, positive control. ${ }^{* * * * *} \mathrm{P}<0.001$ vs. NC. (C) Western blot analysis of BECN1 in MDA-MB-231 cells following sgRNA lentivirus and Cas9 adenovirus infection. GAPDH was used as an internal control. BECN1, Beclin1.

A

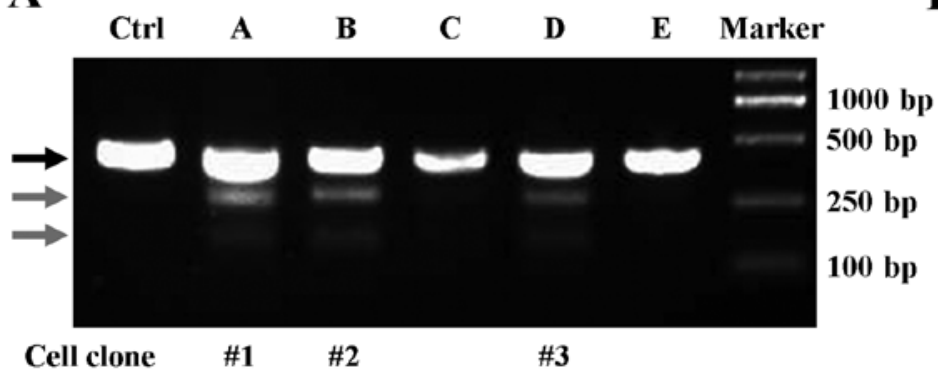

C<smiles>[AsH2][AsH2]</smiles>

B

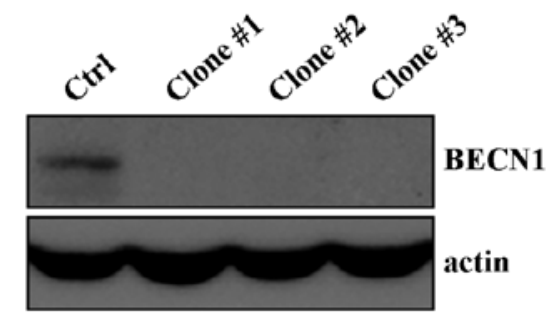

ATGGAAGGGTCTAAGACGTCCAACAACAGCACCATGCAGGTGAGCTTCGTGTGCCA Wild-type

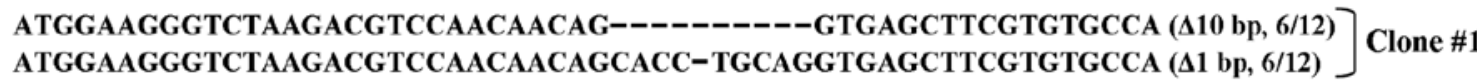

ATGGAAGGGTCTAAGACGTCCAACAACAGCACC-TGCAGGTGAGCTTCGTGTGCCA ( $\triangle 1$ bp, 8/12) ATGGAAGGGTCTAAGACGTCCAACAACAGCACCA-GCAGGTGAGCTTCGTGTGCCA ( $\Delta 1$ bp, 2/12) Clone \#2 ATGGAAGGGTCTAAGACGTCCAACAACAGCA--ATGCAGGTGAGCTTCGTGTGCCA ( $(\Delta 2$ bp, 2/12) ATGGAAGGGTCTAAGACGTCCAACAACAGCACC-TGCAGGTGAGCTTCGTGTGCCA $(\Delta 1$ bp, $2 / 12)$
ATGGAAGGGTCTAAGACGTCCAACAACA-------GCAGGTGAGCTTCGTGTGCCA $(\Delta 7$ bp, 3/12)
ATGGAAGGGTCTAAGACGTCCAACA------------GGTGAGCTTCGTGTGCCA $(\Delta 13$ bp, $7 / 12)]$ Clone \#3

Figure 2. Analysis of BECN1 using a T7EI digestion assay and sequence analysis following BECN1 disruption in MDA-MB-231 cells. (A) T7EI digestion assay. Genomic DNA was extracted from five representative cell clones derived from monoclonal colonies. The editing site was amplified with primer set, B371_F and B793_R, and then subjected to T7EI digestion. The black arrow indicates the wild-type band and the grey arrows indicate mutant bands. Ctrl, wild-type MDA-MB-231 cells. (B) Western blot analysis of BECN1 in wild-type MDA-MB-231 cells (Ctrl) and the selected monoclonal cells, which were named \#1, \#2 and \#3. Actin served as the internal control. (C) Sequencing analysis of the BECN1 gene in the selected clones (\#1, \#2 and \#3). The bracketed number is the PCR clones with a specific edit over the total PCR clones tested. TSS, translation start site. BECN1, Beclin1; T7EI, T7 Endonuclease I. 
A

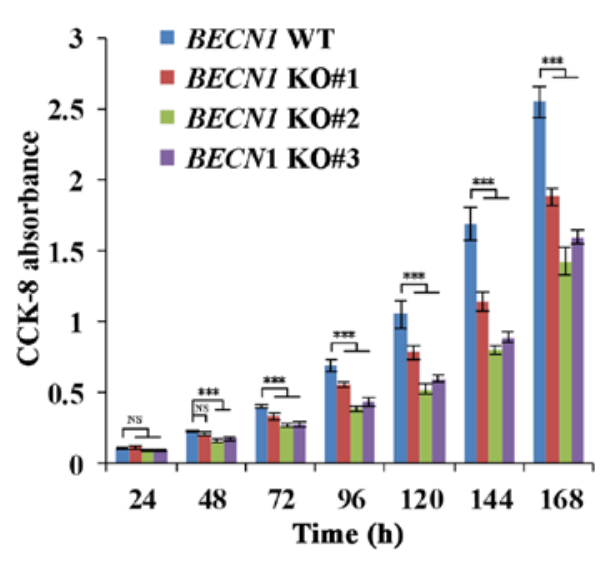

B

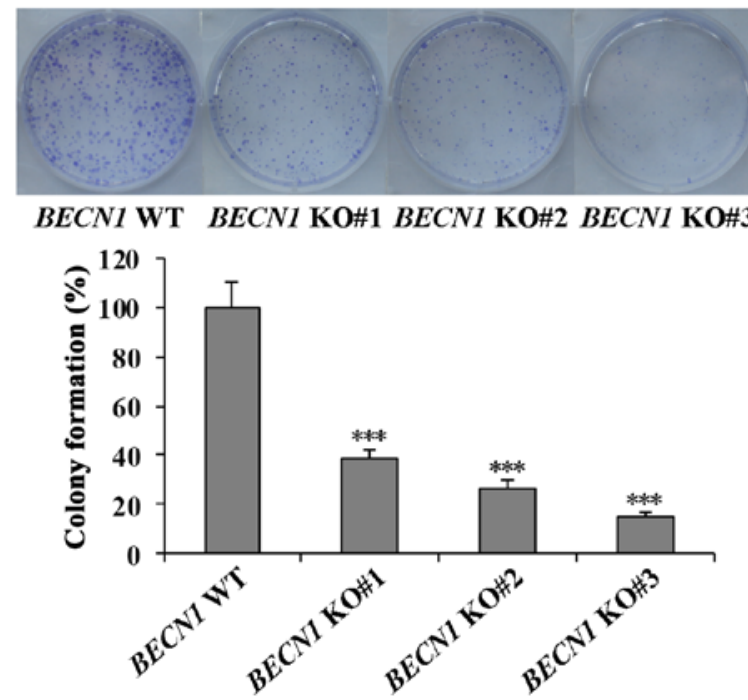

Figure 3. BECN1-knockout suppresses MDA-MB-231 cell growth in vitro. (A) Growth curves of MDA-MB-231 cells following BECN1-knockout. Cellular proliferation was measured using the CCK-8 assay at different time-points. (B) Colony formation of MDA-MB-231 cells following BECN1-knockout. BECN1 WT or KO MDA-MB-231 cells were cultured for up to 2 weeks. Colonies were stained using crystal violet and counted. Quantitative data of colony formation is presented in a histogram (bottom) and the percentage was normalized to WT cells. NS, not significant $(\mathrm{P}>0.05) .{ }^{* * * *} \mathrm{P}<0.001 \mathrm{vs}$. WT cells. BECN1, Beclin1; WT, wild-type; KO, knockout.

A
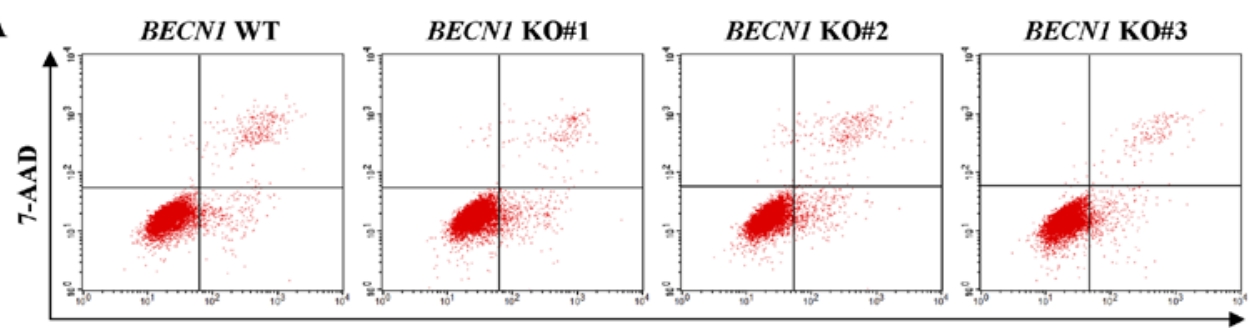

PE Annexin V

\section{B}
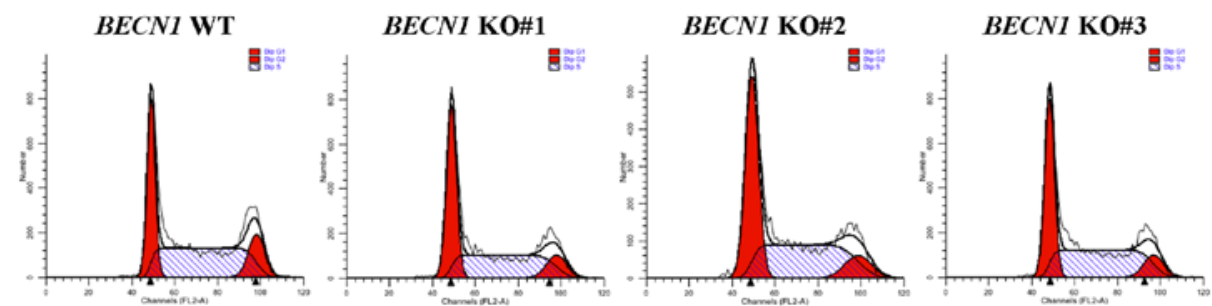

C
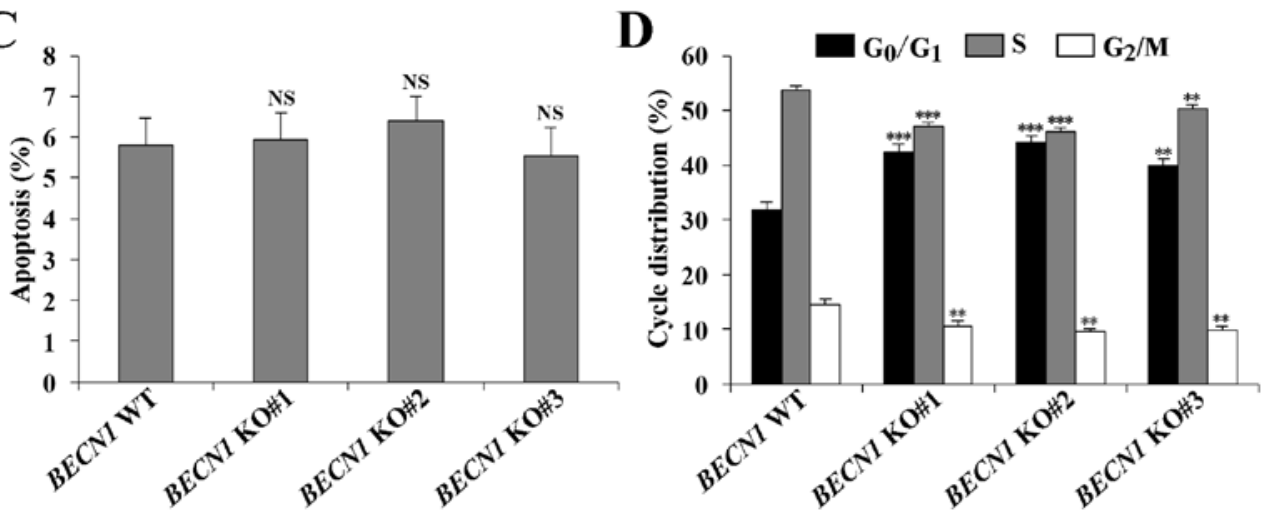

Figure 4. BECN1-knockout induces cell cycle arrest, not apoptosis, in MDA-MB-231 cells. (A) Effect of BECN1-knockout on apoptosis in MDA-MB-231 cells The apoptotic rate was analyzed by Annexin V staining and flow cytometry. The right lower quadrant represents early apoptotic cells, whereas the right upper quadrant contains late apoptotic cells. (B) Effect of BECN1-knockout on cell cycle distribution of MDA-MB-231 cells. Cell cycles were measured by flow cytometry. (C) Quantification of the data shown in A. NS, not significant ( $\mathrm{P}>0.05$ vs. WT cells). (D) Quantification of the data shown in $\mathrm{B}$. ${ }^{* * *} \mathrm{P}<0.01 ;{ }^{* * * *} \mathrm{P}<0.001$ vs. WT cells. BECN1, Beclin1; WT, wild-type; KO, knockout. 
A

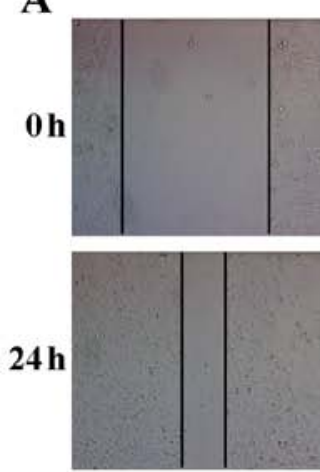

BECN1 WT

B

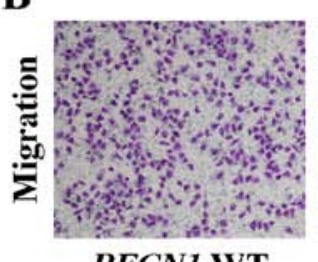

BECN1 WT

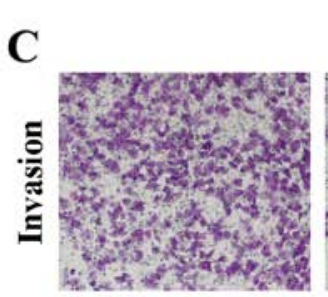

BECN1 WT
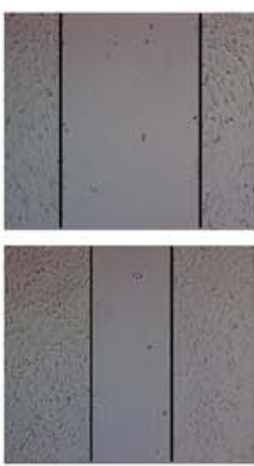

BECN1 KO\#1

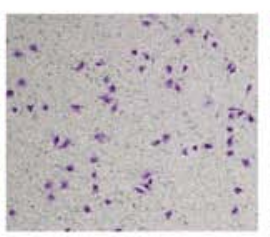

BECN1 KO\#1

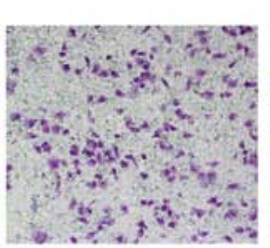

BECN1 KO\#1
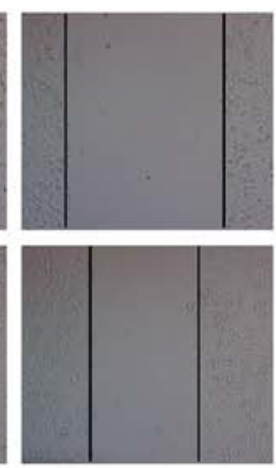

BECN1 KO\#2

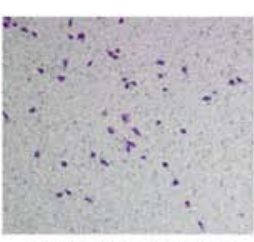

BECN1 KO\#2

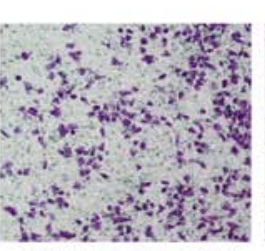

BECN1 KO\#2
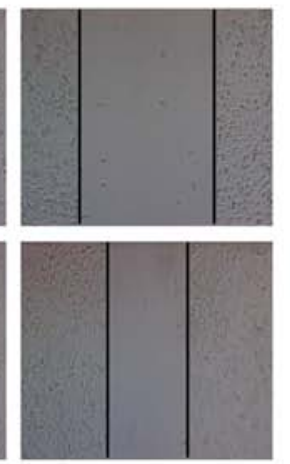

BECN1 KO\#3
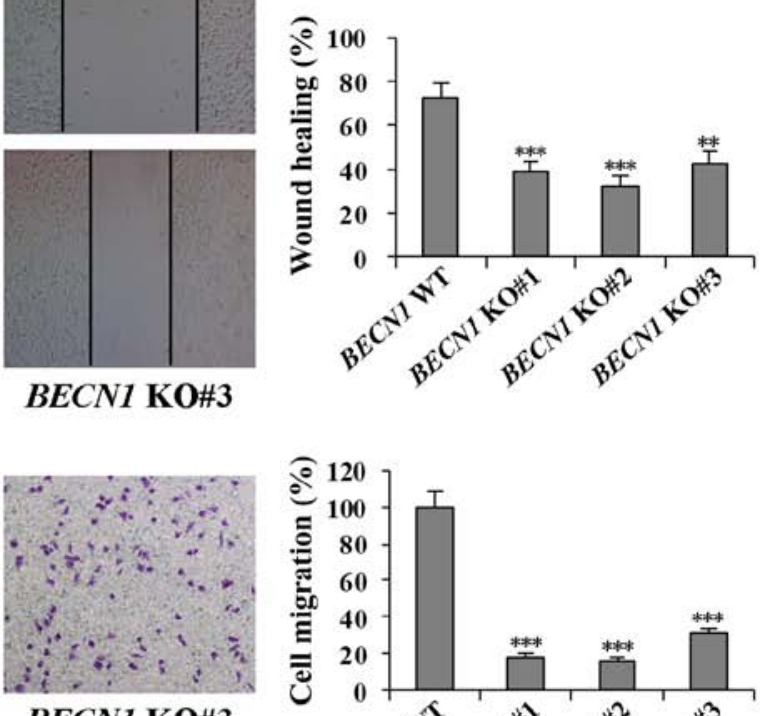

BECN1 KO\#3
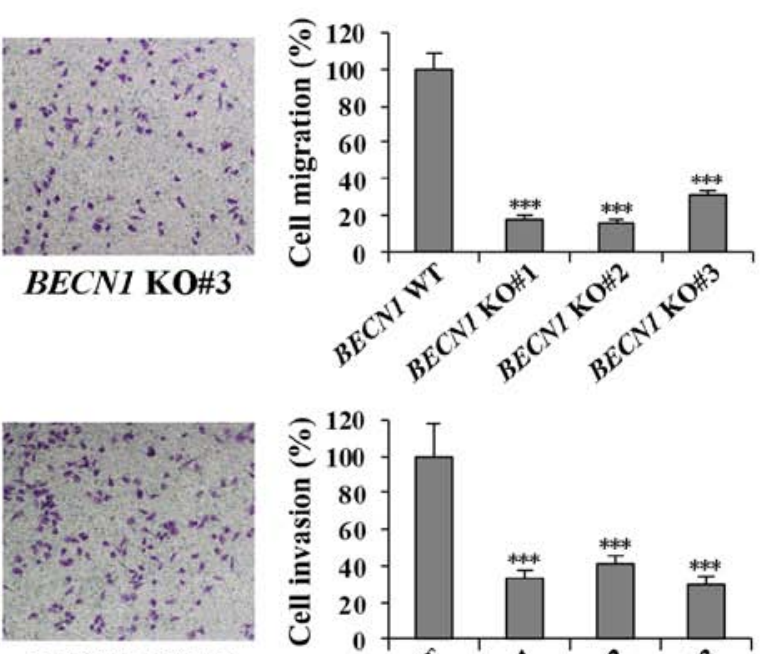

BECN1 KO\#3

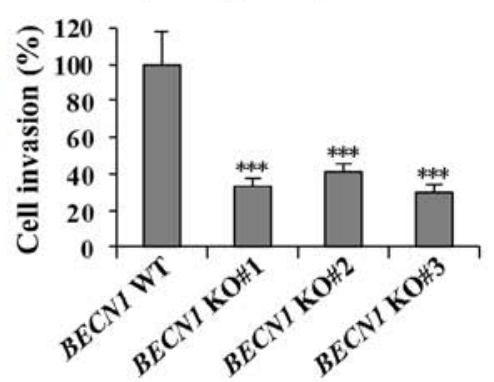

Figure 5. BECN1-knockout inhibits the migratory and invasive ability of MDA-MB-231 cells. (A) Representative images of the wound healing assay (magnification, x100). Right panel, quantitative results. Representative images of the Transwell (B) migration and (C) invasion assays. BECN1 WT or KO MDA-MB-231 cells migrated or invaded to the bottom side of the filter membrane are shown (magnification, x100). Right panel, quantitative results are presented as a histogram. The percentage was normalized to WT cells. Cells were counted in five different randomly selected fields. ${ }^{* *} \mathrm{P}<0.01 ;{ }^{* * *} \mathrm{P}<0.001$ vs. WT cells. $B E C N 1$, Beclin1; WT, wild-type; KO, knockout.

approach to evaluate the ability of a single cell to grow into a colony and essentially tests every cell within a population for its ability to undergo 'unlimited' division (22). It was also determined that BECN1-knockout inhibited the colony formation capacity of MDA-MB-231 cells $(\mathrm{P}<0.001$; Fig. 3B). Therefore, BECN1-knockout MDA-MB-231 cells retained growth capability but exhibited an attenuated proliferation rate.

Next, flow cytometry was performed to investigate whether the anti-proliferative effect of $B E C N 1$-knockout was due to apoptosis or cell cycle arrest. There was no significant difference in apoptosis in the BECN1-knockout MDA-MB-231 cells compared with WT (Fig. 4A and C). However, all three BECN1-knockout cell lines accumulated at the $\mathrm{G}_{0} / \mathrm{G}_{1}$ phase with decreased cell proportions in the $S$ and $G_{2} / M$ phases compared with WT (Fig. 4B and D). The percentage of $\mathrm{G}_{0} / \mathrm{G}_{1}$ cells significantly increased from $31.7 \pm 1.6 \%$ (WT) to $42.5 \pm 1.3 \%(\mathrm{KO} \# 1 ; \mathrm{P}<0.001), 44.1 \pm 1.2 \%(\mathrm{KO \#} 2 ; \mathrm{P}<0.001)$ and $39.9 \pm 1.3 \%(\mathrm{KO \# 3} ; \mathrm{P}<0.01)$. These results suggested that BECN1-mediated autophagy or other pathways serve an important role in cancer cell proliferation via regulation of cell cycle progression.

BECN1-knockout suppresses MDA-MB-231 cell migratory and invasive potential by partially attenuating EMT signals. Subsequently, whether BECN1 affects the tumor metastasis characteristics of migratory and invasive ability in MDA-MB-231 cells was investigated. The wound healing assay revealed that MDA-MB-231 cells with BECN1-knockout exhibited a notably slower scratch closure rate than WT cells (Fig. 5A), which suggested mobility inhibition. The Transwell migration assay further confirmed that BECN1-knockout markedly reduced the migratory ability of MDA-MB-231 cells (Fig. 5B). Furthermore, the invasive potential of BECN1-knockout MDA-MB-231 cells was significantly decreased (Fig. 5C), which was confirmed by the Transwell invasion assay. In brief, the results of the present study demonstrated that BECN1-knockout markedly decreased the migratory and invasive potential of MDA-MB-231 cells in vitro. 
A

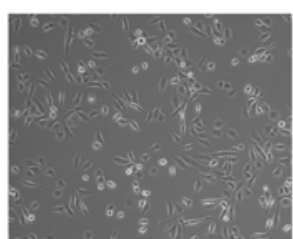

BECN1 WT

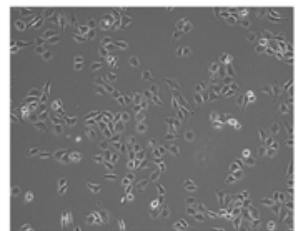

BECN1 KO\#1

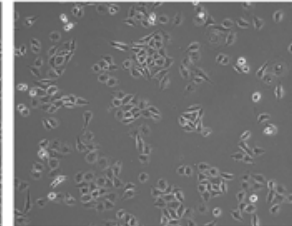

BECN1 KO\#2

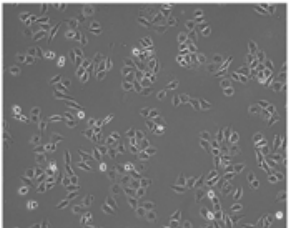

BECN1 KO\#3
B

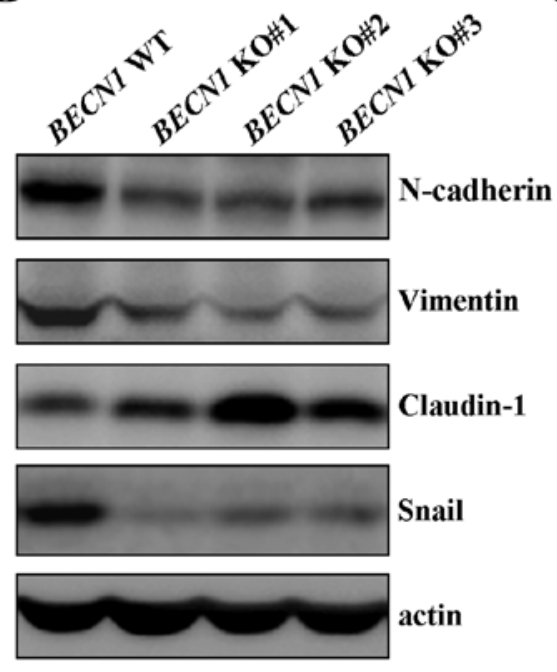

D

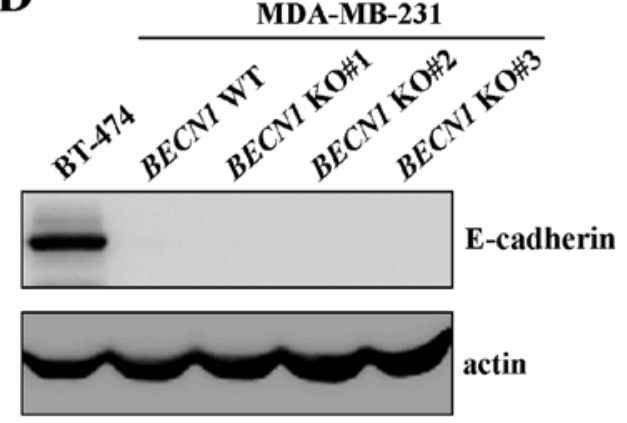

C
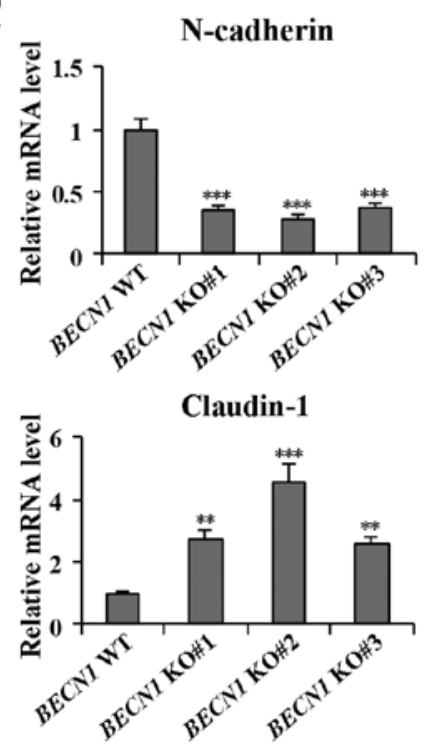

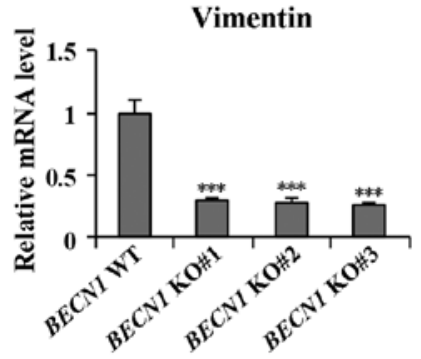

Snail
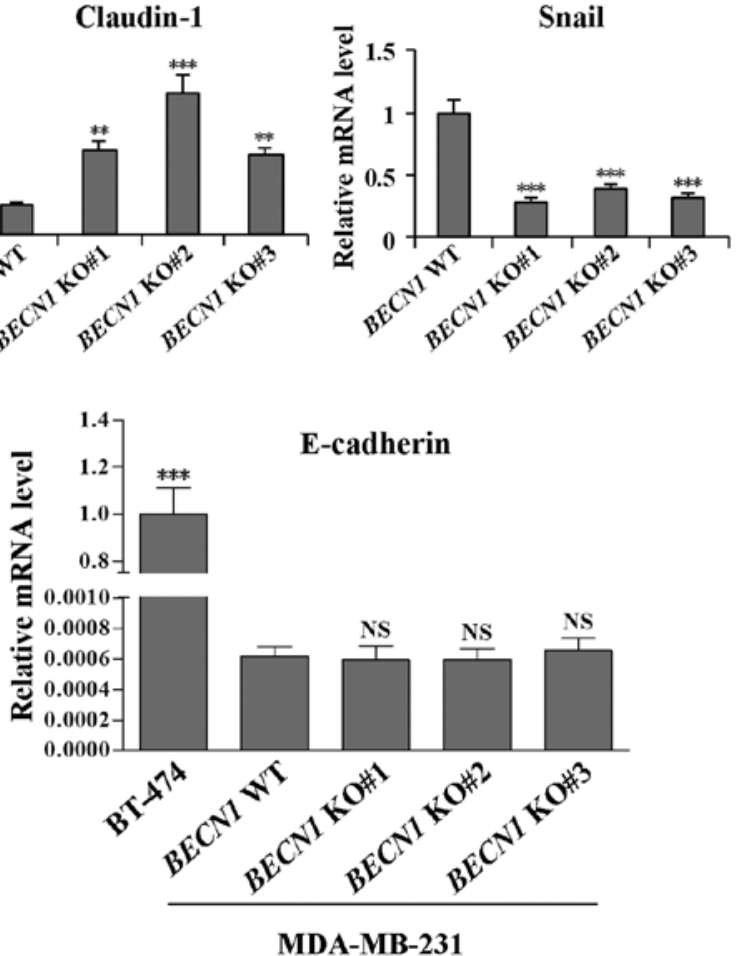

MDA-MB-231

Figure 6. BECN1 partially modulates EMT phenotype in MDA-MB-231 cells. (A) Phase contrast images of $B E C N 1$ WT and KO MDA-MB-231 cells (magnification, x100). (B) Protein levels of tumor EMT markers (N-cadherin, Vimentin, Claudin-1 and Snail) in BECN1 WT and KO MDA-MB-231 cells were detected using western blotting with respective antibodies. Actin served as the internal control. (C) RNA levels of N-cadherin, Vimentin, Claudin-1 and Snail in $B E C N 1$ WT and KO MDA-MB-231 cells were determined using qPCR. The relative mRNA levels were normalized to GAPDH. (D) BECN1-knockout does not affect the expression of E-cadherin in MDA-MB-231 cells. E-cadherin protein was detected using western blotting (left panel) and RNA expression was determined using qPCR (right panel). E-cadherin expression in the epithelial breast cancer BT-474 cell line was used as a positive control. NS, not significant $(\mathrm{P}>0.05) ;{ }^{* *} \mathrm{P}<0.01 ;{ }^{* * *} \mathrm{P}<0.001$ vs. WT MDA-MB-231 cells. qPCR, quantitative polymerase chain reaction; BECN1, Beclin1; EMT, epithelial-mesenchymal transition; WT, wild-type; KO, knockout.

To test whether the effect of $B E C N 1$-knockout on migration and invasion is associated with EMT, the present study assessed the cell morphology under a phase contrast microscope. As demonstrated in Fig. 6A, BECN1-knockout changed the mesenchymal morphology of MDA-MB-231 cells into a partial epithelial-like morphology with decreased cell scattering and increased cell-cell adhesion. Consistent with the morphology changes, expression of mesenchymal markers, including $\mathrm{N}$-cadherin, Vimentin and Snail decreased, while expression of the epithelial marker Claudin-1, but not E-cadherin, increased following BECN1-knockout (Fig. 6B-D), suggesting a partial reversal of EMT. Taken together, the results of the present study demonstrated that $B E C N 1$ regulates breast cancer cell migration and invasion via partial modulation of the EMT signaling pathway.

BECN1 expression is increased in TNBC tissue samples. To determine whether the expression of BECN1 is altered 
A

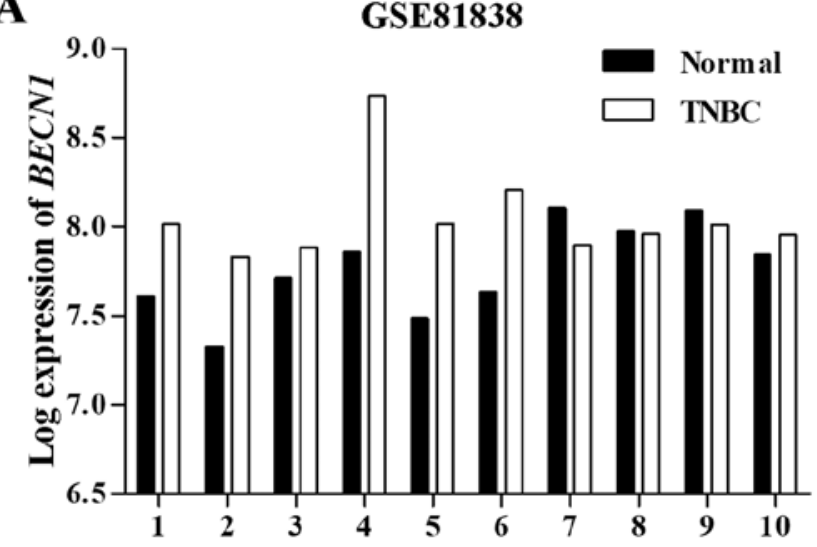

B

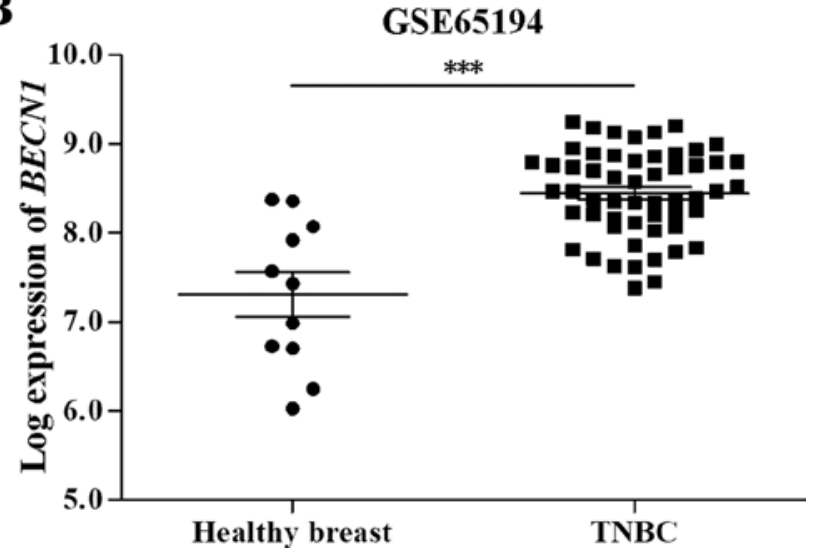

Figure 7. The mRNA expression of BECN1 is upregulated in TNBC tissues. (A) The publicly available GEO dataset, GSE81838, was analyzed to determine $B E C N 1$ expression in TNBC tissues relative to adjacent normal stromal tissues. (B) Analysis of the level of BECN1 in patients with TNBC using the GEO dataset, GSE65194. ${ }^{* * *} \mathrm{P}<0.001$ vs. normal breast sample from mammoplasty surgery. TNBC, triple-negative breast cancer; GEO, Gene Expression Omnibus; $B E C N 1$, Beclin1.

in TNBC tissues, a GEO dataset (GSE81838) containing ten patients with TNBC was analyzed (20). The results demonstrated that BECN1 was significantly upregulated in half of the TNBC tissues compared with adjacent normal stromal tissues (Fig. 7A; $\mathrm{P}<0.05$ ). Meanwhile, another GEO dataset (GSE65194) containing 41 TNBC samples and 11 normal breast tissue samples was analyzed (21). BECN1 expression was significantly increased in tumor tissues compared with healthy breast tissues (Fig. 7B; $\mathrm{P}<0.001$ ). The results suggested that BECN1 may serve as an oncogene in TNBC.

\section{Discussion}

A large volume of data has implied the important role of $B E C N 1$ in autophagy and tumorigenesis. Unfortunately, the embryolethality of Becnl bi-allelic knockout has largely affected further investigation of BECN1 function (9). The function of $B E C N 1$ in tumorigenesis remains controversial, and may be tissue- and context-dependent. While mammary tumor development was either inhibited or unaffected following loss of Palb2, or overexpression of PyMT and $E R B B 2$, monoallelic Becnl loss accelerates mammary tumorigenesis following parity and Wntl-activation (11-13). Fortunately, CRISPR/Cas9-mediated gene disruption at the single cell level shed light on this problem. Using the CRISPR/ Cas9 technique, BECN1 was successfully disrupted in human TNBC MDA-MB-231 cells and screened three monoclonal cells with BECN1-knockout. In a previous study, Aita et al (6) demonstrated that MDA-MB-231 cells have three BECN1 loci. The present study revealed that cell clone \#2 and \#3 harbored three disruptions in BECN1. Clone \#1 only had two versions, which may be due to two loci of BECN1 being the same. In brief, these $B E C N 1$-knockout cell lines provided an ideal cellular model for the investigation into $B E C N 1$ function and demonstrated that BECN1-knockout is not lethal in vitro.

$B E C N 1$ was first identified as a mammalian tumor suppressor by Liang et al (7). BECN1 expression is decreased in human breast epithelial carcinoma cell lines and tissues at the protein level, and induction of BECN1 into MCF-7 cells suppresses proliferation, colony formation in vitro and tumor formation in nude mice (7). However, Gong et al (14) demonstrated that BECN1 is critical for the maintenance of cancer stem-like cells and tumor development in nude mice. Maycotte et al (23) also discovered that knockdown of BECN1 by shRNA inhibits clonogenic proliferation of MDA-MB-231 cells. The present study demonstrated that BECN1 promoted cell proliferation and colony formation in MDA-MB-231 cells. These results and those of previous studies suggested that $B E C N 1$ could function as an oncogene and a tumor suppressor under distinct cellular contexts.

Tumor growth is usually associated with apoptosis and/or cell cycle alterations. Apoptosis is characterized by clear morphological changes, including nuclear condensation and fragmentation, as well as blebbing of the plasma membrane leading to the formation of apoptotic bodies (24). Ectopic overexpression of BECNI does not affect apoptosis under normal culture conditions but inhibits apoptosis induced by starvation, hypoxia and doxorubicin in TNBC cells in vitro (25). Additionally, suppression of BECN1 promotes apoptosis induced by epirubicin $(26)$, tamoxifen $(27,28)$ and lapatinib (29) in breast cancer cells. In the present study, there was no change in the apoptosis of MDA-MB-231 cells following BECN1-knockout. By contrast, BECN1-knockout induced a significant $\mathrm{G}_{0} / \mathrm{G}_{1}$ phase arrest and decreased the proportion of cells in the $S$ and $G_{2} / M$ phases. As a control for cell growth and proliferation, when facing unfavorable conditions or external stress, cells block the cell cycle transiently or irreversibly, which prevents spread of damaged and potentially harmful cells $(30,31)$. A previous study has demonstrated that mRNA and protein expression levels of $B E C N 1$ are upregulated when released from synchronization at the $\mathrm{G}_{1} / \mathrm{S}$ boundary by a double thymidine block in HeLa cells (32). BECN1 is essential for chromosome congression and outer kinetochore assembly (33). Inhibition of BECN1 leads to centrosome amplification (34), which usually causes abnormal cell division and genome instability. These results indicated that BECN1 could affect cancer cell growth by regulating the cell cycle and/or apoptosis depending on the external environment.

Another hallmark of cancer is invasion and metastasis (35). High levels of BECN1 expression are associated 
with more lymph node and distant metastasis in patients with TNBC, and overexpression of BECN1 promotes EMT under starvation conditions in TNBC cells (25). The present study demonstrated that $B E C N 1$-knockout inhibited the migration and invasion of MDA-MB-231 cells. Furthermore, the decreased migratory and invasive ability was associated with a morphological switch in mesenchymal phenotype to a partial epithelial-like phenotype following BECN1-knockout. Upon undergoing EMT, cancer cells convert from a non-invasive epithelial phenotype to an invasive mesenchymal phenotype to obtain migratory and invasive ability, allowing degradation of the extracellular matrix and migration to distant organs (36). Cadherin is a transmembrane adhesion glycoprotein (37) and the switch from E-cadherin to N-cadherin is a remarkable change during EMT (38). In the present study, E-cadherin was not expressed in WT MDA-MB-231 cells, which is consistent with the results of previous studies $(39,40)$; and was not increased following BECN1-knockout, but there was a significant decrease in $\mathrm{N}$-cadherin expression following BECN1-knockout. Another mesenchymal marker is Vimentin, which is associated with cancer invasion and a poor prognosis in various types of cancer (41). In accordance with the change in $\mathrm{N}$-cadherin expression, Vimentin expression was also downregulated following BECN1-knockout. Claudin-1 belongs to the transmembrane protein family, is regarded as the backbone of tight junctions and is associated with tumor metastasis (42). However, the definitive role of Claudin-1 in TNBC is controversial. In patients with TNBC, the Claudin1-negative phenotype predicts a high risk of recurrence and death, which suggested an antitumor function of Claudin-1 (43). However, silencing Claudin-1 expression using shRNA inhibits the migration and invasion of MDA-MB-231 cells (44). In the present study, Claudin-1 expression increased following BECN1-knockout in MDA-MB-231 cells, which may be associated with inhibition of Snail and subsequent transcriptional repression of Claudin-1 (45). Therefore, the decreased migration and invasion capability following $B E C N 1$-knockout could be explained by a partial EMT reversal of MDA-MB-231 cells.

Although previous reports indicated that breast cancer harbors frequent allelic deletions and decreased expression of BECN1 $(6,7,46,47)$, Laddha et al $(48)$ reported that there were no changes in the RNA level of BECN1 in invasive breast carcinoma tissues, compared with normal tissues. The present study demonstrated that BECN1 was upregulated in TNBC tissue samples, compared with adjacent normal stromal tissues and normal breast tissues. Similar expression patterns have been reported in ovarian cancer (49-51), gastric cancer $(52-54)$ and hepatocellular carcinoma $(55,56)$. Correa et al (49) demonstrated that even with prevalent single-copy loss, BECN1 protein expression remained similar in high-grade serous ovarian cancer. BECN1 expression is increased in ovarian cancer compared with benign tumor and normal ovary tissues (50). However, Shen et al (51) observed that expression of BECN1 is reduced in ovarian cancer, compared with benign and borderline ovarian tumors. In addition, Fei et al (54) reported that BECN1 expression in early-stage gastric cancer is significantly higher, while in late-stage cases levels were lower than in adjacent normal tissues (54). These seemingly controversial results may be explained by tumor heterogeneity and the biphasic function of BECN1 in cancer development.

In conclusion, $B E C N 1$-knockout TNBC cell lines were established using the CRISPR/Cas9 system. The results indicated that increased $B E C N 1$ activates numerous properties that are associated with the malignant potential in TNBC cells, including proliferation, colony formation, cell cycle progression, migration, invasion and a partial EMT.

\section{Acknowledgements}

The authors would like to thank Dr Pu-Min Zhang (Beijing Proteome Research Center, Beijing, China) for providing the CRISPR/Cas9 plasmid.

\section{Funding}

The present study was supported by the National Natural Science Foundation of China (grant nos.81372925, 31470897 and 81602457).

\section{Availability of data and materials}

The datasets used and/or analyzed during the current study are available from the corresponding author on reasonable request.

\section{Authors' contributions}

LJZ and PKZ conceived and designed the experiments; CLW, SMZ, LL, SSG, KFF, XDL and YL performed the experiments; CLW, SMZ and LJZ analyzed the data and contributed reagents/materials/analysis tools; CLW, SMZ and PKZ wrote the paper. All authors have read and approved this manuscript.

\section{Ethics approval and consent to participate}

Not applicable.

\section{Patient consent for publication}

Not applicable.

\section{Competing interest}

The authors declare that they have no competing interests.

\section{References}

1. Liang XH, Kleeman LK, Jiang HH, Gordon G, Goldman JE, Berry G, Herman B and Levine B: Protection against fatal Sindbis virus encephalitis by beclin, a novel Bcl-2-interacting protein. J Virol 72: 8586-8596, 1998.

2. Kang R, Zeh HJ, Lotze MT and Tang D: The Beclin 1 network regulates autophagy and apoptosis. Cell Death Differ 18: 571-580, 2011.

3. Funderburk SF, Wang QJ and Yue Z: The Beclin 1-VPS34 complex--at the crossroads of autophagy and beyond. Trends Cell Biol 20: 355-362, 2010.

4. Russell RC, Tian Y, Yuan H, Park HW, Chang YY, Kim J, Kim H, Neufeld TP, Dillin A and Guan KL: ULK1 induces autophagy by phosphorylating Beclin-1 and activating VPS34 lipid kinase. Nat Cell Biol 15: 741-750, 2013. 
5. Wang RC, Wei Y, An Z, Zou Z, Xiao G, Bhagat G, White M, Reichelt J and Levine B: Akt-mediated regulation of autophagy and tumorigenesis through Beclin 1 phosphorylation. Science 338: 956-959, 2012

6. Aita VM, Liang XH, Murty VV, Pincus DL, Yu W, Cayanis E, Kalachikov S, Gilliam TC and Levine B: Cloning and genomic organization of beclin 1, a candidate tumor suppressor gene on chromosome 17q21. Genomics 59: 59-65, 1999.

7. Liang XH, Jackson S, Seaman M, Brown K, Kempkes B, Hibshoosh $\mathrm{H}$ and Levine B: Induction of autophagy and inhibition of tumorigenesis by beclin 1. Nature 402: 672-676, 1999.

8. Qu X, Yu J, Bhagat G, Furuya N, Hibshoosh H, Troxel A, Rosen J, Eskelinen EL, Mizushima N, Ohsumi Y, et al: Promotion of tumorigenesis by heterozygous disruption of the beclin 1 autophagy gene. J Clin Invest 112: 1809-1820, 2003.

9. Yue Z, Jin S, Yang C, Levine AJ and Heintz N: Beclin 1, an autophagy gene essential for early embryonic development, is a haploinsufficient tumor suppressor. Proc Natl Acad Sci USA 100: 15077-15082, 2003

10. He Y, Zhao X, Subahan NR, Fan L, Gao J and Chen H: The prognostic value of autophagy-related markers beclin-1 and microtubule-associated protein light chain 3B in cancers: $\mathrm{A}$ systematic review and meta-analysis. Tumour Biol 35: 7317-7326, 2014.

11. Cicchini M, Chakrabarti R, Kongara S, Price S, Nahar R, Lozy F, Zhong H, Vazquez A, Kang Y and Karantza V: Autophagy regulator BECN1 suppresses mammary tumorigenesis driven by WNT1 activation and following parity. Autophagy 10 : 2036-2052, 2014.

12. Lozy F, Cai-McRae X, Teplova I, Price S, Reddy A, Bhanot G, Ganesan S, Vazquez A and Karantza V: ERBB2 overexpression suppresses stress-induced autophagy and renders ERBB2induced mammary tumorigenesis independent of monoallelic Becn1 loss. Autophagy 10: 662-676, 2014.

13. Huo Y, Cai H, Teplova I, Bowman-Colin C, Chen G, Price S, Barnard N, Ganesan S, Karantza V, White E, et al: Autophagy opposes p53-mediated tumor barrier to facilitate tumorigenesis in a model of PALB2-associated hereditary breast cancer. Cancer Discov 3: 894-907, 2013.

14. Gong C, Bauvy C, Tonelli G, Yue W, Delomenie C, Nicolas V, Zhu Y, Domergue V, Marin-Esteban V, Tharinger $\mathrm{H}$, et al: Beclin 1 and autophagy are required for the tumorigenicity of breast cancer stem-like/progenitor cells. Oncogene 32 : 2261-2272, 2272e 2261-2211, 2013.

15. Cong L, Ran FA, Cox D, Lin S, Barretto R, Habib N, Hsu PD, Wu X, Jiang W, Marraffini LA, et al: Multiplex genome engineering using CRISPR/Cas systems. Science 339: 819-823, 2013.

16. Wright AV, Nuñez JK and Doudna JA: Biology and applications of CRISPR Systems: Harnessing nature's toolbox for genome engineering. Cell 164: 29-44, 2016.

17. Sánchez-Rivera FJ and Jacks T: Applications of the CRISPR-Cas9 system in cancer biology. Nat Rev Cancer 15: 387-395, 2015.

18. Shalem O, Sanjana NE and Zhang F: High-throughput functional genomics using CRISPR-Cas9. Nat Rev Genet 16: 299-311, 2015.

19. Livak KJ and Schmittgen TD: Analysis of relative gene expression data using real-time quantitative PCR and the 2(-Delta Delta C(T)) Method. Methods 25: 402-408, 2001

20. Lehmann BD, Jovanović B, Chen X, Estrada MV, Johnson KN Shyr Y, Moses HL, Sanders ME and Pietenpol JA: Refinement of triple-negative breast cancer molecular subtypes: Implications for neoadjuvant chemotherapy selection. PLoS One 11: e0157368, 2016.

21. Maire V, Némati F, Richardson M, Vincent-Salomon A, Tesson B, Rigaill G, Gravier E, Marty-Prouvost B, De Koning L, Lang G, et al: Polo-like kinase 1: A potential therapeutic option in combination with conventional chemotherapy for the management of patients with triple-negative breast cancer. Cancer Res 73: 813-823, 2013

22. Franken NA, Rodermond HM, Stap J, Haveman J and van Bree C: Clonogenic assay of cells in vitro. Nat Protoc 1: 2315-2319, 2006.

23. Maycotte P, Gearheart CM, Barnard R, Aryal S, Mulcahy Levy JM, Fosmire SP, Hansen RJ, Morgan MJ, Porter CC, Gustafson DL, et al: STAT3-mediated autophagy dependence identifies subtypes of breast cancer where autophagy inhibition can be efficacious. Cancer Res 74: 2579-2590, 2014.

24. Mariño G, Niso-Santano M, Baehrecke EH and Kroemer G: Self-consumption: The interplay of autophagy and apoptosis. Nat Rev Mol Cell Biol 15: 81-94, 2014.
25. Wang MC, Wu AG, Huang YZ, Shao GL, Ji SF, Wang RW, Yuan HJ, Fan XL, Zheng LH and Jiao QL: Autophagic regulation of cell growth by altered expression of Beclin 1 in triple-negative breast cancer. Int J Clin Exp Med 8: 7049-7058, 2015.

26. Sun WL, Chen J, Wang YP and Zheng H: Autophagy protects breast cancer cells from epirubicin-induced apoptosis and facilitates epirubicin-resistance development. Autophagy 7: 1035-1044, 2011.

27. Qadir MA, Kwok B, Dragowska WH, To KH, Le D, Bally MB and Gorski SM: Macroautophagy inhibition sensitizes tamoxifen-resistant breast cancer cells and enhances mitochondrial depolarization. Breast Cancer Res Treat 112: 389-403, 2008.

28. Gu Y, Chen T, Li G, Xu C, Xu Z, Zhang J, He K, Zheng L, Guan Z, Su X, et al: Lower Beclin 1 downregulates HER2 expression to enhance tamoxifen sensitivity and predicts a favorable outcome for ER positive breast cancer. Oncotarget 8: 52156-52177, 2016.

29. Han J, Hou W, Lu C, Goldstein LA, Stolz DB, Watkins SC and Rabinowich H: Interaction between Her2 and Beclin-1 proteins underlies a new mechanism of reciprocal regulation. J Biol Chem 288: 20315-20325, 2013

30. Kohrman AQ and Matus DQ: Divide or conquer: Cell cycle regulation of invasive behavior. Trends Cell Biol 27: 12-25, 2017.

31. Malumbres $M$ and Barbacid M: Cell cycle, CDKs and cancer: A changing paradigm. Nat Rev Cancer 9: 153-166, 2009.

32. Li Z, Ji X, Wang D, Liu J and Zhang X: Autophagic flux is highly active in early mitosis and differentially regulated throughout the cell cycle. Oncotarget 7: 39705-39718, 2016.

33. Frémont S, Gérard A, Galloux M, Janvier K, Karess RE and Berlioz-Torrent C: Beclin-1 is required for chromosome congression and proper outer kinetochore assembly. EMBO Rep 14: 364-372, 2013

34. Park JM, Tougeron D, Huang S, Okamoto K and Sinicrope FA: Beclin 1 and UVRAG confer protection from radiation-induced DNA damage and maintain centrosome stability in colorectal cancer cells. PLoS One 9: e100819, 2014.

35. Hanahan D and Weinberg RA: Hallmarks of cancer: The next generation. Cell 144: 646-674, 2011.

36. Jiang JH, Liu C, Cheng H, Lu Y, Qin Y, Xu YF, Xu J, Long J, Liu L, Ni QX, et al: Epithelial-mesenchymal transition in pancreatic cancer: Is it a clinically significant factor? Biochim Biophys Acta 1855: 43-49, 2015.

37. Gumbiner BM: Cell adhesion: The molecular basis of tissue architecture and morphogenesis. Cell 84: 345-357, 1996.

38. Peng Z, Wang CX, Fang EH, Wang GB and Tong Q: Role of epithelial-mesenchymal transition in gastric cancer initiation and progression. World J Gastroenterol 20: 5403-5410, 2014

39. Liu CY, Lin HH, Tang MJ and Wang YK: Vimentin contributes to epithelial-mesenchymal transition cancer cell mechanics by mediating cytoskeletal organization and focal adhesion maturation. Oncotarget 6: 15966-15983, 2015.

40. Fukagawa A, Ishii H, Miyazawa K and Saitoh M: $\delta E F 1$ associates with DNMT1 and maintains DNA methylation of the E-cadherin promoter in breast cancer cells. Cancer Med 4: 125-135, 2015.

41. Satelli A and Li S: Vimentin in cancer and its potential as a molecular target for cancer therapy. Cell Mol Life Sci 68: 3033-3046, 2011

42. Ding L, Lu Z, Lu Q and Chen YH: The claudin family of proteins in human malignancy: A clinical perspective. Cancer Manag Res 5: 367-375, 2013

43. Ma F, Ding X, Fan Y, Ying J, Zheng S, Lu N and Xu B: A CLDN1-negative phenotype predicts poor prognosis in triplenegative breast cancer. PLoS One 9: e112765, 2014

44. Zhao X, Zou Y, Gu Q, Zhao G, Gray H, Pfeffer LM and Yue J: Lentiviral vector mediated Claudin1 silencing inhibits epithelial to mesenchymal transition in breast cancer cells. Viruses 7 : 2965-2979, 2015

45. Martínez-Estrada OM, Cullerés A, Soriano FX, Peinado H, Bolós V, Martínez FO, Reina M, Cano A, Fabre M and Vilaró S: The transcription factors Slug and Snail act as repressors of Claudin-1 expression in epithelial cells. Biochem J 394: 449-457, 2006.

46. Li Z, Chen B, Wu Y, Jin F, Xia Y and Liu X: Genetic and epigenetic silencing of the beclin 1 gene in sporadic breast tumors. BMC Cancer 10: 98, 2010.

47. Yao Q, Chen J, Lv Y, Wang T, Zhang J, Fan J and Wang L: The significance of expression of autophagy-related gene Beclin, Bcl-2, and Bax in breast cancer tissues. Tumour Biol 32: 1163-1171, 2011.

48. Laddha SV, Ganesan S, Chan CS and White E: Mutational landscape of the essential autophagy gene BECN1 in human cancers. Mol Cancer Res 12: 485-490, 2014. 
49. Correa RJ, Valdes YR, Shepherd TG and DiMattia GE: Beclin-1 expression is retained in high-grade serous ovarian cancer yet is not essential for autophagy induction in vitro. J Ovarian Res 8: 52, 2015.

50. Zhao Y, Chen S, Gou WF, Xiao LJ, Takano Y and Zheng HC: Aberrant Beclin 1 expression is closely linked to carcinogenesis, differentiation, progression, and prognosis of ovarian epithelial carcinoma. Tumour Biol 35: 1955-1964, 2014.

51. Shen Y, Li DD, Wang LL, Deng R and Zhu XF: Decreased expression of autophagy-related proteins in malignant epithelial ovarian cancer. Autophagy 4: 1067-1068, 2008.

52. Yu M, Gou WF, Zhao S, Xiao LJ, Mao XY, Xing YN, Takahashi H, Takano Y and Zheng HC: Beclin 1 expression is an independent prognostic factor for gastric carcinomas. Tumour Biol 34: 1071-1083, 2013.
53. Yu S, Li G, Wang Z, Wang Z, Chen C, Cai S and He Y: Low expression of MAP1LC3B, associated with low Beclin-1, predicts lymph node metastasis and poor prognosis of gastric cancer. Tumour Biol 37: 15007-15017, 2016.

54. Fei B, Ji F, Chen X, Liu Z, Li S, Mo Z and Fang X: Expression and clinical significance of Beclin-1 in gastric cancer tissues of various clinical stages. Oncol Lett 11: 2271-2277, 2016.

55. Song H, Xia SL, Liao C, Li YL, Wang YF, Li TP and Zhao MJ: Genes encoding Pir51, Beclin 1, RbAp48 and aldolase b are up or down-regulated in human primary hepatocellular carcinoma. World J Gastroenterol 10: 509-513, 2004.

56. Al-Shenawy HA: Expression of Beclin-1, an autophagy-related marker, in chronic hepatitis and hepatocellular carcinoma and its relation with apoptotic markers. APMIS 124: 229-237, 2016. 